\title{
Disentangling Scrambling and Decoherence via Quantum Teleportation
}

\author{
Beni Yoshida ${ }^{1}$ and Norman Y. Yao ${ }^{2,3}$ \\ ${ }^{1}$ Perimeter Institute for Theoretical Physics, Waterloo, Ontario N2L 2Y5, Canada \\ ${ }^{2}$ Department of Physics, University of California Berkeley, Berkeley, California 94720, USA \\ ${ }^{3}$ Materials Sciences Division, Lawrence Berkeley National Laboratory, Berkeley, California 94720, USA
}

(Received 23 July 2018; revised manuscript received 9 November 2018; published 9 January 2019)

\begin{abstract}
Out-of-time-order correlation (OTOC) functions provide a powerful theoretical tool for diagnosing chaos and the scrambling of information in strongly interacting, quantum systems. However, their direct and unambiguous experimental measurement remains an essential challenge. At its core, this challenge arises from the fact that the effects of both decoherence and experimental noise can mimic that of information scrambling, leading to decay of OTOCs. Here, we analyze a quantum teleportation protocol that explicitly enables one to differentiate between scrambling and decoherence. Moreover, we demonstrate that within this protocol, one can extract a precise "noise" parameter which quantitatively captures the nonscramblinginduced decay of OTOCs. Using this parameter, we prove explicit bounds on the true value of the OTOC. Our results open the door to experimentally measuring quantum scrambling with built-in verifiability.
\end{abstract}

DOI: 10.1103/PhysRevX.9.011006

\section{INTRODUCTION}

The thermalization of strongly interacting systems causes information about the initial configuration to become "scrambled" at late times, wherein two initial states (with the same conserved quantities) become indistinguishable without measuring a macroscopic number of observables [1-4]. Recent studies on the dynamics of such information scrambling have sharpened our understanding of chaos in quantum many-body systems [5-27], and have led to new insights on a variety of questions ranging from the black-hole information paradox $[5,11,15,28,29]$ to transport phenomena in non-Fermi liquids [30,31]. While a precise definition of quantum scrambling remains elusive, a powerful proxy for characterizing its behavior is provided by out-of-time-order correlation (OTOC) functions, which take the general form $\langle V(0) W(t) V(0) W(t)\rangle$, where $V, W$ are operators that act on sufficiently small subsystems $[8,10,12,32]$. The intuition behind this correlator is an attempt to measure the influence of one observable at earlier times on another observable at later times-in essence, a quantum version of the so-called butterfly effect. To do this, however, requires the precise reversal of time evolution and thus, poses a daunting challenge for any experiment.

Published by the American Physical Society under the terms of the Creative Commons Attribution 4.0 International license. Further distribution of this work must maintain attribution to the author(s) and the published article's title, journal citation, and DOI.
Subject Areas: Atomic and Molecular Physics,

Quantum Physics, Quantum Information
Despite this challenge, a tremendous amount of interest has been devoted to the development of protocols [33-35] and platforms [36,37] for the direct measurement of OTOCs. The crucial difficulty in interpreting such measurements can be summarized as follows: For a generic interacting system without symmetries, the scrambling of quantum information will cause out-of-time-order correlation functions to decay to zero. However, both decoherence and imperfect experimental controls (e.g., time reversal)

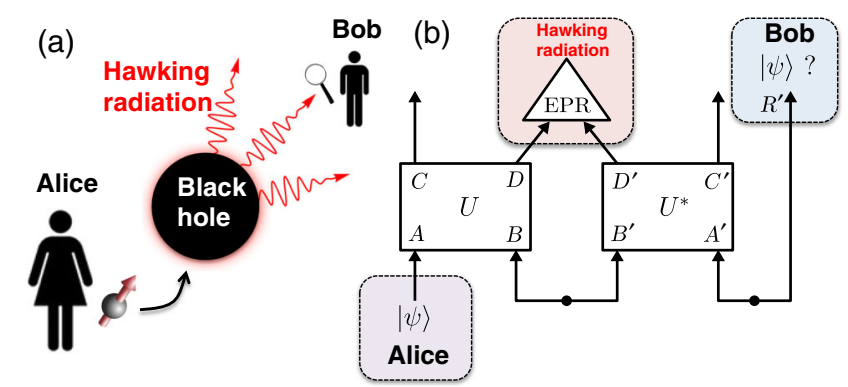

FIG. 1. (a) In the Hayden-Preskill variant of the black-hole decoding problem [5], one asks whether Bob can decode the state of Alice's quantum spin using only Hawking radiation and an entangled partner of the black hole. Any successful "decoding" serves as affirmation for the existence of scrambling dynamics. (b) For an arbitrary unitary $U$, one can utilize a teleportation-based decoding protocol to probe the scrambling behavior of the circuit [29]. Crucially, even in the presence of arbitrary noise and imperfections, the teleportation fidelity acts as a metric for quantum scrambling and enables the bounding of the mutual information between Alice's and Bob's reference quantum registers. 
will also cause OTOCs to decay to zero. At present, the only way to distinguish between these two contributionsnamely, true chaotic scrambling versus noise and decoherence-is to perform full quantum tomography on the many-body system, requiring exponentially many measurements in the number of qubits [38-41]. To this end, the ability to distinguish between genuine quantum information scrambling and extrinsic decoherence remains an essential open question [42].

In this paper, we analyze a quantum teleportation protocol that explicitly enables such differentiation. We present three main results. First, we demonstrate that within our protocol, one can extract a "noise" parameter, which quantifies the nonscrambling-induced decay of OTOCs. Here, we focus on two illustrative examples: (i) depolarization (i.e., a nonunitary error) and (ii) imperfect "backwards" time evolution (i.e., a unitary error). Second, using this noise parameter, we provide a bound on the true scrambling-induced decay of the OTOC. Again, we analyze two cases, one which applies specifically to the situation of unitary errors and another which applies to arbitrary errors. Finally, we describe two simple realizations of our protocol amenable to near-term, intermediate scale qubit and qutrit systems as well as their generalizations to include Grover search [43].

The essence of our approach is based upon a recent decoding algorithm for the Hayden-Preskill variant of the black-hole information problem [5,29]. The connection between this decoding algorithm and information scrambling can be understood as follows: If the dynamics of a black hole are unitary, then one should, in principle, be able to retrieve a quantum state that is thrown in from the Hawking radiation that comes out [Fig. 1(a)]. Crucially, it turns out that such a successful "decoding" of the original quantum state serves as smoking-gun evidence for the existence of true scrambling dynamics.

\section{ORGANIZATION AND SUMMARY OF MAIN RESULTS}

In Sec. III, we begin by reviewing the information theoretic interpretation of scrambling and OTOCs. Then, using the example of a depolarizing quantum channel, we illustrate the fact that decoherence can result in the decay of OTOCs even in the absence of scrambling dynamics. This allows us to propose a sharp measure which quantifies the ratio of scrambling-induced versus decoherence-induced OTOC decay. Moreover, it reveals that the genuine metric for scrambling should be taken as the mutual information between subsystems and not simply the measured OTOC. The groundwork being laid, in Sec. IV, we introduce the teleportation-based decoding protocol and clarify its operation in the ideal case without noise and decoherence. Then in Sec. V, we turn to an analysis of the protocol in the presence of arbitrary noise and decoherence. Here, we demonstrate that the protocol provides a quantitative

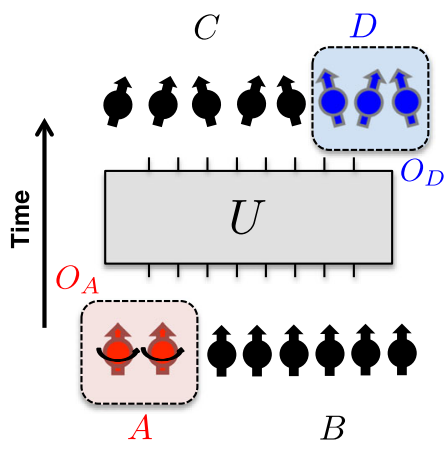

FIG. 2. Schematic image of the setup associated with an out-oftime-ordered correlation function, $\left\langle O_{A}(0) O_{D}(t) O_{A}(0) O_{D}(t)\right\rangle$. Time runs upward and evolution is generated by the unitary $U$. The Hilbert space decomposes as $\mathcal{H}=\mathcal{H}_{A} \otimes \mathcal{H}_{B}=\mathcal{H}_{C} \otimes \mathcal{H}_{D}$. Ideally, operators $O_{A}$ and $O_{D}$ act on sufficiently small subsystems of the full system.

estimate for the amount of dissipation in the system. In Sec. VI, motivated by recent experiments, we restrict ourselves to a subclass of noise and imperfections, with a focus on coherent errors. Under this restriction, we show that one can explicitly bound the ideal value of the OTOC (i.e., in the absence of errors), using the experimentally measured value of the OTOC. In Sec. VII, we generalize such a bound to the case of arbitrary errors and prove that one can utilize the teleportation fidelity to bound the mutual information between subsystems (and hence the amount of scrambling). Finally, in Sec. VIII, we propose and analyze two experimental implementations of our protocol in nearterm intermediate scale quantum simulators. We focus on a class of Clifford scramblers that saturate the lower bound for OTOCs. In Sec. IX, we offer some concluding remarks and intriguing directions to be pursued.

\section{CHARACTERIZING SCRAMBLING AND DECOHERENCE}

\section{A. Definition of scrambling in terms of OTOCs}

Let us begin by providing a definition for quantum scrambling in terms of the behavior of out-of-time-order correlation functions $[16,29]$ :

$$
\begin{aligned}
\left\langle O_{X} O_{Y}(t) O_{Z} O_{W}(t)\right\rangle & \\
\approx & \left\langle O_{X} O_{Z}\right\rangle\left\langle O_{Y}\right\rangle\left\langle O_{W}\right\rangle+\left\langle O_{X}\right\rangle\left\langle O_{Z}\right\rangle\left\langle O_{Y} O_{W}\right\rangle \\
& \quad-\left\langle O_{X}\right\rangle\left\langle O_{Y}\right\rangle\left\langle O_{Z}\right\rangle\left\langle O_{W}\right\rangle,
\end{aligned}
$$

where $O_{X}, O_{Z}$ are operators that act on subsystem $A$ (at time zero) and $O_{Y}, O_{W}$ are operators that act on subsystem $D$ (at time $t$ ), as depicted in Fig. 2. This equation becomes exact in the thermodynamic limit for chaotic systems at late times and can also be derived from the eigenstate thermalization hypothesis $[1-4,44]$. While we will focus on infinite temperature systems with $\rho=(1 / d) \mathbb{I}$, we note that this 
definition naturally generalizes to finite temperatures. Our above definition of scrambling is required to hold for all local operators, but a slightly more coarse-grained characterization of scrambling (and one which is easier to probe experimentally) can be achieved via the averaged OTOC [15]:

$$
\overline{\langle\mathrm{OTOC}\rangle} \equiv \iint d O_{A} d O_{D}\left\langle O_{A} O_{D}(t) O_{A}^{\dagger} O_{D}^{\dagger}(t)\right\rangle
$$

where $\int d O_{R}$ is the Haar average over all unitary operators on subsystem $R$. This Haar integral can be replaced by an average over Pauli operators:

$$
\begin{aligned}
& \iint d O_{A} d O_{D}\left\langle O_{A} O_{D}(t) O_{A}^{\dagger} O_{D}^{\dagger}(t)\right\rangle \\
& \quad=\frac{1}{d_{A}^{2} d_{D}^{2}} \sum_{P_{A}, P_{D}}\left\langle P_{A} P_{D}(t) P_{A}^{\dagger} P_{D}^{\dagger}(t)\right\rangle,
\end{aligned}
$$

where $P_{A(D)}$ are Pauli operators and $d_{A(D)}$ is the dimension of the subsystem [45]. Working at infinite temperature and using Eq. (1) then yields the scrambled value of the averaged OTOC as [48]

$$
\overline{\langle\mathrm{OTOC}}_{S} \approx \frac{1}{d_{A}^{2}}+\frac{1}{d_{D}^{2}}-\frac{1}{d_{A}^{2} d_{D}^{2}}
$$

This scrambled value $\overline{\langle\mathrm{OTOC}}_{S}$ is achieved for a Haar random unitary as $d \rightarrow \infty$ [15]. On the other hand, for arbitrary unitary time evolution, $\overline{\langle\mathrm{OTOC}\rangle}$ is bounded from above by unity and from below by $\max \left[\left(1 / d_{A}^{2}\right),\left(1 / d_{D}^{2}\right)\right]$; the fact that it never fully decays to zero is because it contains contributions from cases where $P_{A}=\mathbb{I}$ or $P_{D}=\mathbb{I}$. We note that the minimal scrambled value is only asymptotically achieved for large systems with $d \gg d_{A} \gg d_{D}$ or $d \gg d_{D} \gg d_{A}$ [49].

\section{B. Decoding as a route to scrambling}

In order to characterize the effect of decoherence on the averaged OTOC, it will be useful to first recall the information theoretic interpretation of $\overline{\langle\mathrm{OTOC}\rangle}$ in terms of the mutual information between subsystems. To do so, we will utilize the so-called state representation of the time-evolution operator $U[5,15]$. This representation allows us to view a unitary operator $U$, acting on an $n$ qubit Hilbert space $\mathcal{H}_{A B}$, as a pure quantum state, supported on a $2 n$-qubit Hilbert space $\mathcal{H}_{A B} \otimes \mathcal{H}_{R B^{\prime}}\left(\simeq \mathcal{H}_{R C D B^{\prime}}\right)$ :

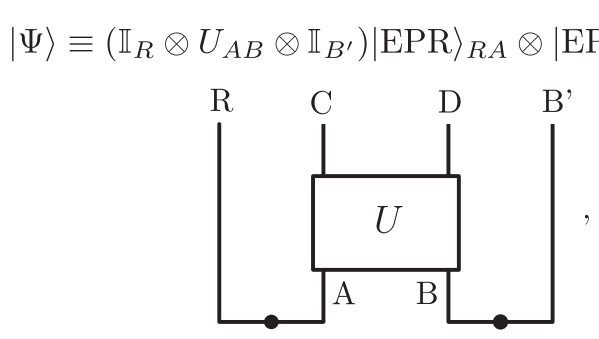

where time runs upward and the horizontal lines in the diagram represent Einstein-Podolsky-Rosen (EPR) pairs $\left(|\mathrm{EPR}\rangle \equiv(1 / \sqrt{d}) \sum_{j=1}^{d}|j\rangle \otimes|j\rangle\right)$, while the dots capture the $1 / \sqrt{d}$ normalization factor in the EPR pair. Crucially, this representation allows us to characterize the scrambling behavior of the time evolution $U$ via the entanglement properties of the pure state $|\Psi\rangle$.

Three remarks are in order. First, for noninteracting time evolutions, including free-fermion dynamics or SWAP operators, $|\Psi\rangle$ contains mostly bipartite entanglement among subsystems. On the other hand, for strongly interacting time evolutions that lead to scrambling, $|\Psi\rangle$ consists of multipartite entanglement delocalized over the full Hilbert space $R C D B^{\prime}$.

Second, we note that $|\Psi\rangle$ is precisely the state of interest in the Hayden-Preskill thought experiment [5]. In particular, the Hilbert spaces $A, B, C, D$ [Eq. (5)] support, respectively, Alice's input states, the initial black hole, the remaining black hole, and the Hawking radiation. Meanwhile, $R$ serves as a reference for Alice's input state, while $B^{\prime}$ is the entangled partner of the black hole. The Hayden-Preskill decoding problem can then be stated as follows: when can Bob decode Alice's quantum state using only the Hawking radiation $D$ and the entangled black-hole partner $B^{\prime}[5]$ ? The answer, somewhat naturally, is when the (von Neumann) mutual information between $R$ and $B^{\prime} D$ is maximal. More precisely, when this is the case, there exists a unitary operator acting on $B^{\prime} D$ which distills an EPR pair between $R$ and $B^{\prime} D$ with high fidelity, thereby faithful recovering Alice's input state $[5,50]$.

Third, we note that for maximally mixed states the Rényi-2 mutual information, $I^{(2)}\left(R, B^{\prime} D\right)$, lower bounds the von Neumann mutual information, $I\left(R, B^{\prime} D\right)$ [51]. This is particularly useful since $I^{(2)}\left(R, B^{\prime} D\right)$ is, in fact, directly related to our previously defined averaged OTOC $[15,16,23]$ :

$$
\overline{\langle\mathrm{OTOC}\rangle}=2^{-I^{(2)}\left(R, B^{\prime} D\right)},
$$

where $I^{(2)}\left(R, B^{\prime} D\right) \equiv S_{R}^{(2)}+S_{B^{\prime} D}^{(2)}-S_{R B^{\prime} D}^{(2)}$ and the Rényi-2 entropies $S^{(2)}$ are evaluated with respect to the state $|\Psi\rangle$. To this end, in an ideal (noiseless) system, the smallness of $\overline{\langle\mathrm{OTOC}\rangle}$, which characterizes the amount of scrambling in the system, is also sufficient to diagnose Bob's faithful recovery of Alice's state [52]. 
However, the essential point is that in a system with noise and imperfections, the smallness of $\overline{\langle\mathrm{OTOC}\rangle}$ can result either from decoherence or from true scrambling behavior. Crucially, only the latter will contribute to Bob's ability to decode Alice's quantum state. In the following subsections, we will first focus on identifying the effects of decoherence on the averaged OTOC. With this in hand, we will then provide a precise metric to distinguish between decoherence and scrambling.

\section{Effects of decoherence on the OTOC}

To understand the effects of decoherence, let us consider the following quantum channel $\mathcal{Q}$ :

$$
\rho \rightarrow \mathcal{Q}(\rho)=(1-p) U \rho U^{\dagger}+p \frac{\mathbb{I}}{d} \operatorname{Tr}(\rho),
$$

which suffers from depolarization with probability $p$ [53]. For traceless operators, one finds that the out-of-time-order correlators behave as

$\left\langle O_{X} \widetilde{O_{Y}}(t) O_{Z} \widetilde{O_{W}}(t)\right\rangle=(1-p)^{2}\left\langle O_{X} O_{Y}(t) O_{Z} O_{W}(t)\right\rangle$,

where we use the tilde to indicate observables time evolved under the quantum channel $\mathcal{Q}$, while time-evolved operators without a tilde are evolved under the unitary portion of the channel $U$ [54]. Thus, even in the absence of information scrambling [i.e., in the actual behavior of $\left.\left\langle O_{X} O_{Y}(t) O_{Z} O_{W}(t)\right\rangle\right]$, the measured OTOCs for the channel $\mathcal{Q}$ can become small owing to decoherence; in particular, undergoing depolarization with a finite probability per unit time induces an exponential decay of the measured values of OTOCs.

The difference between scrambling and decoherence can be further sharpened and made precise by considering the late-time asymptotics of OTOCs, which serve as our operational definition of quantum scrambling in Eq. (1). Specifically, under a completely depolarizing channel (e.g., $p=1$ ), the out-of-time-order correlators decompose as follows:

$$
\left\langle O_{X} O_{Y}(t) O_{Z} O_{W}(t)\right\rangle=\left\langle O_{X} O_{Z}\right\rangle\left\langle O_{Y}\right\rangle\left\langle O_{W}\right\rangle,
$$

which contains only the first term in Eq. (1).

As before, one can also examine the averaged OTOCs associated with the channel $\mathcal{Q}$ :

$$
\langle\widetilde{\mathrm{OTOC}}\rangle \equiv \iint d O_{A} d O_{D}\left\langle O_{A} \widetilde{O_{D}}(t) \widetilde{O_{A}^{\dagger}} \widetilde{O_{D}^{\dagger}}(t)\right\rangle .
$$

Note that for an arbitrary quantum channel, the value of $\langle\widetilde{\text { OTOC}}\rangle$ is now lower bounded by $1 / d_{D}^{2}$, whereas in the absence of imperfections, $\langle\overline{\mathrm{OTOC}}\rangle$ was previously lower bounded by $\max \left[\left(1 / d_{A}^{2}\right),\left(1 / d_{D}^{2}\right)\right]$.

In the above discussion, we have implicitly assumed that both $O_{Y}$ and $O_{W}$ are evolved with the same (possibly imperfect) quantum channel $\mathcal{Q}$. However, it is certainly of interest to consider the situation where they evolve under two different quantum channels, which is precisely the experimental scenario if one performs backwards time evolution imperfectly. We will address this case in detail a bit later.

\section{Distinguishing decoherence from scrambling}

As we have shown, for an arbitrary quantum channel, the decay of OTOCs is not sufficient to experimentally diagnose the scrambling behavior of the system. To this end, we now provide a formal metric for distinguishing between scrambling and decoherence in noisy quantum systems. Let us consider the state representation of the channel $\mathcal{Q}$ defined as follows:

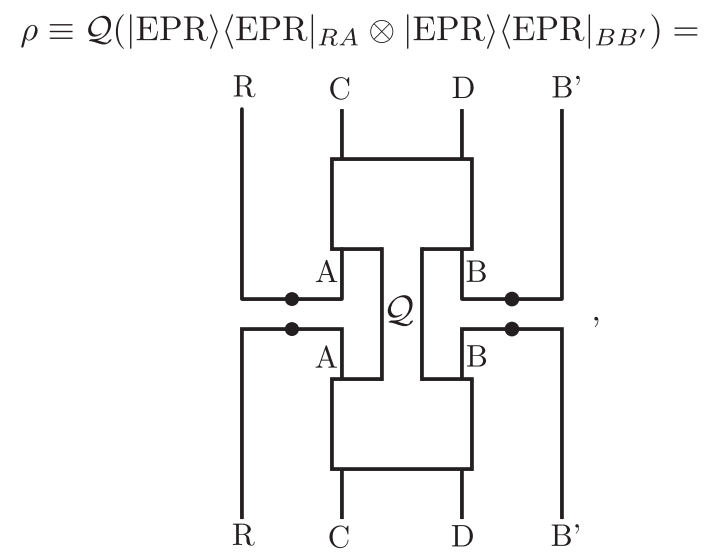

where $\rho$ is the system's density matrix.

To gain some intuition, let us consider the two limiting cases: $p=0$ (no decoherence) and $p=1$ (full depolarization). In the first case, $\mathcal{Q}$ is purely unitary and can be decomposed into two separate boxes corresponding to $U, U^{\dagger}$, wherein $\rho=|\Psi\rangle\langle\Psi|$ is a pure state with $|\Psi\rangle$ as defined in Eq. (5). In the second case, $\mathcal{Q}$ induces complete depolarization and the corresponding quantum state $\rho$ is a maximally mixed state on $R C D B^{\prime}$ with graphical representation:

$$
\rho=\frac{1}{d^{2}} \mathbb{I}_{R} \otimes \mathbb{I}_{C} \otimes \mathbb{I}_{D} \otimes \mathbb{I}_{B^{\prime}}=\left.\left.\oint_{\mathrm{R}}^{\mathrm{R}} \oint_{\mathrm{C}}^{\mathrm{C}}\right|_{\mathrm{D}} ^{\mathrm{D}}\right|_{\mathrm{B}^{\prime}} ^{\mathrm{D}}
$$

As one can see from this example, for nonunitary time evolution, $\rho$ is not a pure state.

However, one nevertheless finds that the averaged OTOC can be re-expressed in terms of Rényi-2 entropies evaluated with respect to $\rho$ [55]:

$$
\langle\widetilde{\mathrm{OTOC}}\rangle=2^{-\left(S_{B^{\prime} D}^{(2)}+S_{D}^{(2)}-S_{B^{\prime}}^{(2)}\right)} .
$$


The astute reader may wonder why this looks quite similar to the aforementioned result in the ideal, noiseless case [Eq. (6)]. Since $S_{R}^{(2)}+S_{B^{\prime}}^{(2)}=S_{C}^{(2)}+S_{D}^{(2)}=n$ (where $n$ is the total number of qubits in $R B^{\prime}$ ), if $\rho$ was in fact a pure state, then one would have $S_{R B^{\prime} D}^{(2)}=S_{C}^{(2)}$, and hence,

$$
S_{B^{\prime} D}^{(2)}+S_{D}^{(2)}-S_{B^{\prime}}^{(2)}=I^{(2)}\left(R, B^{\prime} D\right)
$$

Thus, when $\mathcal{Q}$ is unitary (e.g., when the depolarizing probability $p=0$ ), the averaged OTOC indeed reduces to our previous result for the ideal system [Eq. (6)].

Crucially, for a generic noisy quantum channel, the state $\rho$ is not pure and $S_{B^{\prime} D}^{(2)}+S_{D}^{(2)}-S_{B^{\prime}}^{(2)} \neq I^{(2)}\left(R, B^{\prime} D\right)$. Herein lies the essence of our result: The genuine metric for scrambling, the mutual information, is not directly measured via the OTOC, which instead only measures the entropy, $S_{B^{\prime} D}^{(2)}+S_{D}^{(2)}-S_{B^{\prime}}^{(2)}$.

The deviation between these two quantities serves as a natural metric or "noise parameter" capturing the decoherence present in the channel $\mathcal{Q}$ :

$$
\delta \equiv \frac{2^{I^{(2)}\left(R, B^{\prime} D\right)}}{2^{S_{B^{\prime} D}^{(2)}+S_{D}^{(2)}-S_{B^{\prime}}^{(2)}}}=2^{S_{C}^{(2)}-S_{R B^{\prime} D}^{(2)}},
$$

where $\delta=1$ for unitary time evolution while $\delta=1 / d_{D}^{2}$ for a completely depolarizing channel. Note that for any $\delta<1$, one knows that decoherence is at least partially responsible for the observed decay in the averaged OTOC. More succinctly, there are two physical mechanisms that cause $\langle\widetilde{\text { OTOC}}\rangle$ to decay. First, entangling $B^{\prime} D$ with $R$ (as per unitary scrambling) and, second, entangling $B^{\prime} D$ with the environment (as in a depolarizing channel); $\delta$ captures the ratio between these two contributions.

In the following sections, we will turn to the experimental measurement and characterization of $\delta$, via a quantum-teleportation-based decoding protocol $[5,29]$. In Sec. IV, we will begin by setting up the framework of the protocol in the ideal case (decoherence and noise free), while in Secs. V and VI, we will shift our attention to investigate a variety of imperfections (i.e., both unitary and nonunitary errors).

\section{TELEPORTATION-BASED DECODING PROTOCOL (IDEAL CASE)}

\section{A. Representing the OTOC as a thermofield double state}

To begin, let us consider the diagrammatic representation of the OTOC in the case of unitary time evolution $U$ :

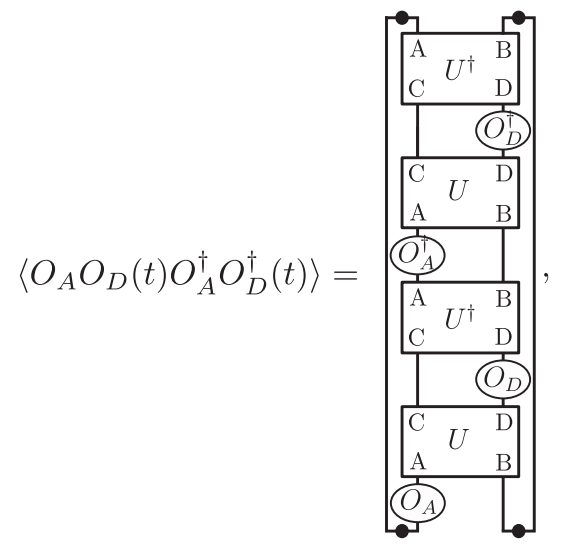

where again, time runs upward and the expectation value is taken at infinite temperature; in our diagrammatic representation, connecting the legs of the input and output corresponds to taking a trace with respect to a maximally mixed state. While the OTOC, $\left\langle O_{A} O_{D}(t) O_{A}^{\dagger} O_{D}^{\dagger}(t)\right\rangle$, is defined on the Hilbert space $\mathcal{H}_{A B}$, it can be recast as the expectation value of local operators on the doubled Hilbert space, $\mathcal{H}_{A B} \otimes \mathcal{H}_{B^{\prime} A^{\prime}}$. In particular, consider the following state, $\quad\left|\Phi_{O_{A}}\right\rangle \equiv\left(U_{A B} \otimes U_{B^{\prime} A^{\prime}}^{*}\right)\left(O_{A} \otimes \mathbb{I}_{B B^{\prime} A^{\prime}}\right)|\mathrm{EPR}\rangle_{A B B^{\prime} A^{\prime}}$, which lives in $\mathcal{H}_{A B} \otimes \mathcal{H}_{B^{\prime} A^{\prime}}$. This is the so-called thermofield double state (at infinite temperature) perturbed by local operator $O_{A}$ and then time evolved by $U \otimes U^{*}$. Taking the expectation value of $\mathbb{I}_{C} \otimes O_{D} \otimes O_{D}^{*} \otimes \mathbb{I}_{C^{\prime}}$ in this state results in

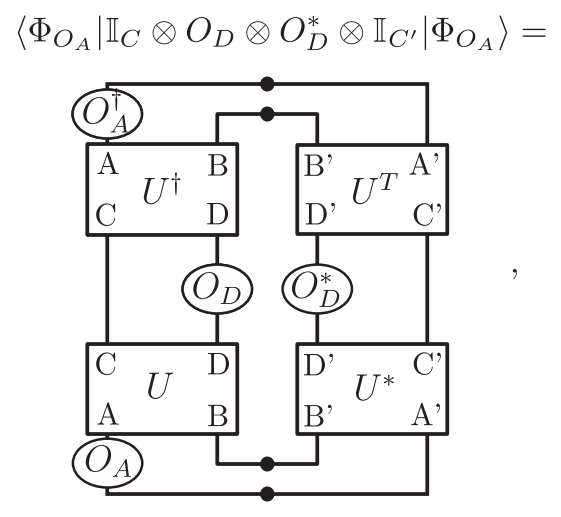

which is exactly equivalent to the OTOC defined in Eq. (16). This equivalence is most easily seen by "unfolding" the diagram of Eq. (16) while noting that $(U \otimes \mathbb{I})|\mathrm{EPR}\rangle=$ $\left(\mathbb{I} \otimes U^{T}\right)|\mathrm{EPR}\rangle$, or in diagrammatic form,

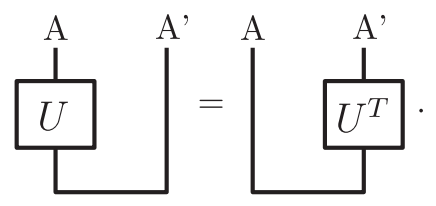

Since $\quad\left\langle O_{A} O_{D}(t) O_{A}^{\dagger} O_{D}^{\dagger}(t)\right\rangle=\left\langle\Phi_{O_{A}}\right| \mathbb{I}_{C} \otimes O_{D} \otimes O_{D}^{*} \otimes$ $\mathbb{I}_{C^{\prime}}\left|\Phi_{O_{A}}\right\rangle$, one can directly measure OTOCs as an expectation 
value of $O_{D} \otimes O_{D}^{*}$ in the doubled Hilbert space $\mathcal{H}_{A B} \otimes \mathcal{H}_{B^{\prime} A^{\prime}}$; then to compute $\overline{\langle\mathrm{OTOC}\rangle}$, one can simply average over the various operators, $O_{A}, O_{D}$.

As aforementioned, a more elegant and efficient method for measuring $\overline{\langle\mathrm{OTOC}\rangle}$ has recently emerged in the form of a probabilistic decoding protocol (via postselected teleportation) for the Hayden-Preskill thought experiment [29].

\section{B. Decoding protocol in the ideal case}

In the decoding protocol, in addition to Alice's reference state, Bob also prepares an additional EPR pair $|\mathrm{EPR}\rangle_{A^{\prime} R^{\prime}}$ before applying $U^{*}$ to both the entangled black-hole partner $B^{\prime}$ and the $A^{\prime}$ part of his EPR pair. In order to decode Alice's state, Bob must create an EPR pair between Alice's reference state $R$ and his remaining register qubit $R^{\prime}$. After time evolution, the system is in the state

$$
\begin{aligned}
\left|\Psi_{\mathrm{in}}\right\rangle= & \left(\mathbb{I}_{R} \otimes U_{A B} \otimes U_{B^{\prime} A^{\prime}}^{*} \otimes \mathbb{I}_{R^{\prime}}\right) \\
& |\mathrm{EPR}\rangle_{R A} \otimes|\mathrm{EPR}\rangle_{B B^{\prime}} \otimes|\mathrm{EPR}\rangle_{A^{\prime} R^{\prime}} \\
= & l^{\mathrm{R}}
\end{aligned}
$$

Next, Bob collects pairs of qubits on $D D^{\prime}$ and performs a projective measurement onto $|\mathrm{EPR}\rangle_{D D^{\prime}}$, resulting in the state

$$
\left|\Psi_{\text {out }}\right\rangle=\frac{1}{\sqrt{P_{\mathrm{EPR}}}} \mathbb{I}_{R C} \otimes \Pi_{D D^{\prime}} \otimes \mathbb{I}_{C^{\prime} R^{\prime}}\left|\Psi_{\mathrm{in}}\right\rangle=
$$

where $P_{\mathrm{EPR}}$ represents the probability of measuring $|\mathrm{EPR}\rangle_{D D^{\prime}}$. Noting that $\left\langle\Psi_{\text {out }} \mid \Psi_{\text {out }}\right\rangle=\left(1 / P_{\mathrm{EPR}}\right)\left\langle\Psi_{\text {in }}\right| \mathbb{I}_{R C} \otimes$ $\Pi_{D D^{\prime}} \otimes \mathbb{I}_{C^{\prime} R^{\prime}}\left|\Psi_{\text {in }}\right\rangle=1$ yields the diagram for $P_{\mathrm{EPR}}$ :

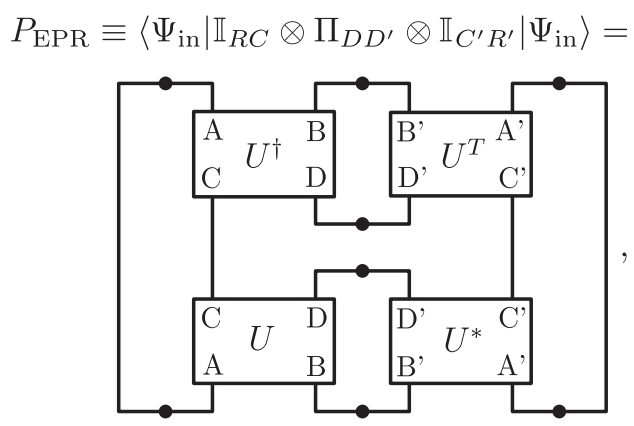

where $\Pi_{D D^{\prime}}=|\mathrm{EPR}\rangle\left\langle\left.\mathrm{EPR}\right|_{D D^{\prime}}\right.$ represents the projective measurement. The fidelity of Bob's decoding (of Alice's state) can then be computed via the EPR projection fidelity on $R R^{\prime}$ :

$$
F_{\mathrm{EPR}} \equiv\left\langle\Psi_{\text {out }}\left|\mathbb{I}_{C D D^{\prime} C^{\prime}} \otimes \Pi_{R R^{\prime}}\right| \Psi_{\text {out }}\right\rangle
$$

It has been shown [29] that if the time evolution $U$ is scrambling an EPR pair, $|\mathrm{EPR}\rangle_{R R^{\prime}}$, can be distilled with high fidelity by postselecting the measurement result on $|\mathrm{EPR}\rangle_{D D^{\prime}}$. Thus, the projection $\Pi_{D D^{\prime}}$ not only serves to decouple Bob's register $R^{\prime}$ from the remaining black holes $C C^{\prime}$, but also teleports Alice's quantum state $|\psi\rangle$ to Bob's register [Fig. 1(b)].

In the ideal, noiseless case, this probabilistic decoding protocol enables one to measure the averaged OTOC associated with $U$ in two different ways, using the values of $P_{\mathrm{EPR}}$ and $F_{\mathrm{EPR}}$, respectively. First, noting that $\int d O_{D} O_{D} \otimes O_{D}^{*}=\Pi_{D D^{\prime}}$, one finds via a simple graphical derivation that [16]

$$
P_{\mathrm{EPR}}=\overline{\langle\mathrm{OTOC}\rangle} .
$$

Thus, by keeping track of the probability associated with the projective measurement $\Pi_{D D^{\prime}}$, one directly measures the averaged OTOC. In the case of $F_{\mathrm{EPR}}$, one can use the following equation:

$$
\begin{aligned}
P_{\mathrm{EPR}} F_{\mathrm{EPR}} & =\left\langle\Psi_{\mathrm{in}}\left|\Pi_{R R^{\prime}} \Pi_{D D^{\prime}} \otimes \mathbb{I}_{C C^{\prime}}\right| \Psi_{\mathrm{in}}\right\rangle \\
& =\frac{1}{d_{A}^{2}} \\
& =\frac{1}{d_{A}^{2}}
\end{aligned}
$$

to derive

$$
F_{\mathrm{EPR}}=\frac{1}{d_{A}^{2} \overline{\langle\mathrm{OTOC}\rangle}} .
$$

To this end, the teleportation fidelity of Alice's state into Bob's register also directly encodes the averaged OTOC.

While both $P_{\mathrm{EPR}}$ and $F_{\mathrm{EPR}}$ measure $\langle\overline{\mathrm{OTOC}}\rangle$, there is an important (but subtle) distinction from the perspective of experiments; in particular, for a scrambling unitary, the former becomes small while the latter becomes large. Thus, when using $P_{\mathrm{EPR}}$, an experiment cannot distinguish 
between a decay in signal arising from scrambling or decoherence. On the other hand, when using $F_{\mathrm{EPR}}$, since decoherence can never enhance the fidelity, a successful decoding always serves as a definite signature of quantum scrambling. This difference will become more apparent in Secs. V and VI when we explicitly consider the effects of noise and decoherence.

\section{Teleportation of a quantum state}

In the previous subsection, we formulated the decoding protocol in terms of the distillation of EPR pairs on $R R^{\prime}$. This formulation implicitly assumes an average over Alice's input state $|\psi\rangle$. However, in the context of experiments, one necessarily performs the teleportation protocol for individual quantum states. Moreover, for dynamics that are not fully scrambling, the dependence of the decoding fidelity on the initial state can be used to discern certain properties of the unitary. An example of this is provided by a system evolving under classical random dynamics, where teleportation only occurs for computational basis states.

To this end, we now consider the decoding protocol for a specific input wave function, where Alice prepares $|\psi\rangle$ on $A$, and Bob checks to see if he obtains $|\psi\rangle$ on $R^{\prime}$ [Fig. 1(b)]:

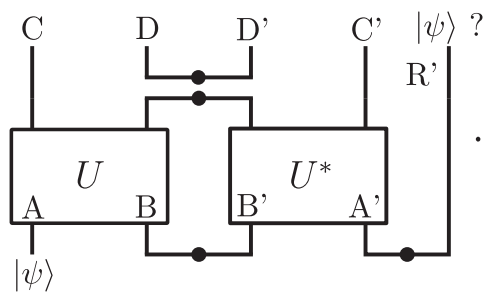

Interestingly, such a setup for decoding specific states can probe more fine-grained properties of OTOCs. The probability of measuring an EPR pair on $D D^{\prime}$ is given by

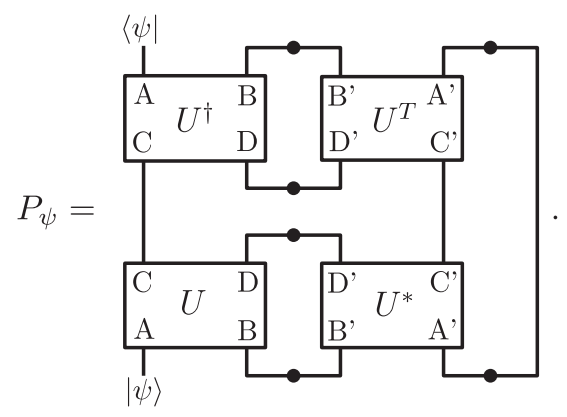

This probability can be reexpressed in terms of OTOCs as follows:

$$
P_{\psi}=\iint d O_{D} d \phi\left\langle O_{A} O_{D}(t) O_{A}^{\dagger} O_{D}^{\dagger}(t)\right\rangle,
$$

where $O_{A}=|\psi\rangle\langle\phi|$ and the average over $O_{A}$ is performed by integrating over $|\phi\rangle$. It suffices to take an average over any set of orthogonal states (i.e., $\{|0\rangle,|1\rangle,|2\rangle, \ldots\}$ ), since the above Haar integral involves only the first moment of $|\phi\rangle$. By inserting an EPR projection onto $C C^{\prime}$, one arrives at the following lower bound:

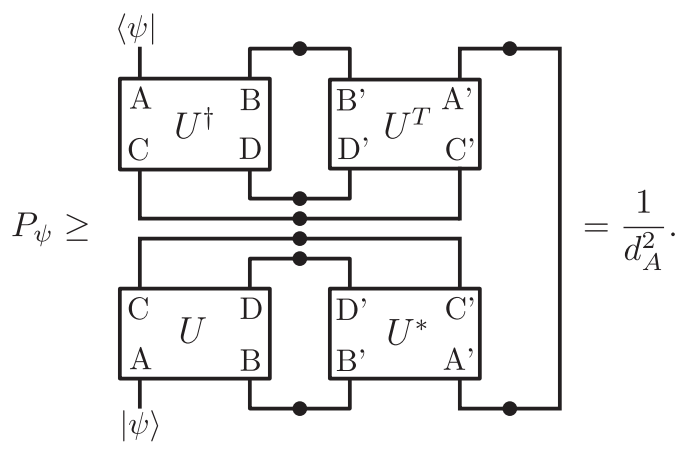

To recover $P_{\mathrm{EPR}}$, one simply averages over (orthogonal) states, $P_{\mathrm{EPR}}=\int d \psi P_{\psi}$. Since the minimal value of $P_{\mathrm{EPR}}$ is also $1 / d_{A}^{2}$, this minimum is achieved when $P_{\psi}=\left(1 / d_{A}^{2}\right)$ for all states. Letting $F_{\psi}$ be the decoding fidelity after postselection, one finds

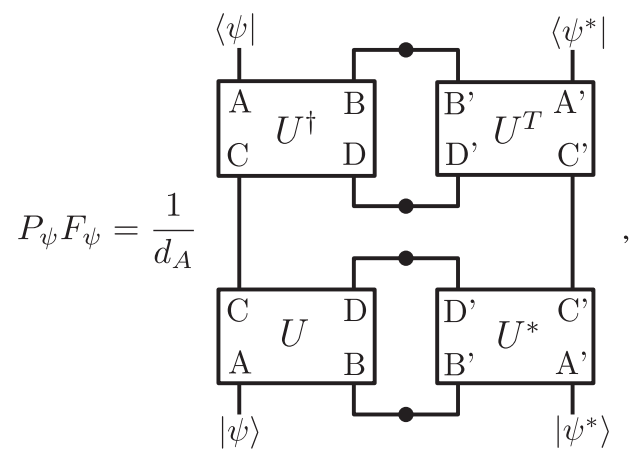

leading to the bound [56]:

$$
P_{\psi} F_{\psi} \geq \frac{1}{d_{A}^{2}} \Rightarrow F_{\psi} \geq \frac{1}{d_{A}^{2} P_{\psi}} .
$$

Thus, in the ideal case, a small value of $P_{\psi}$ guarantees the faithful postselected teleportation of $|\psi\rangle$ from Alice to Bob. In contrast to the previous subsection, we note that the value of $P_{\psi} F_{\psi}$ depends on the initial state $|\psi\rangle$.

One can also recast $P_{\psi} F_{\psi}$ as an OTOC,

$$
P_{\psi} F_{\psi}=\frac{1}{d_{A}} \int d O_{D}\left\langle O_{A} O_{D}(t) O_{A}^{\dagger} O_{D}^{\dagger}(t)\right\rangle,
$$

where $O_{A}=|\psi\rangle\langle\psi|$. Then, by averaging over input states [57], one obtains

$$
\int d \psi P_{\psi} F_{\psi}=\frac{1}{d_{A}+1}\left(P_{\mathrm{EPR}}+\frac{1}{d_{A}}\right)
$$

for the ideal, noise-free case. 


\section{Physical interpretation of EPR projection}

Interestingly, Eq. (32) suggests that all of the accessible information about OTOCs probed in a state decoding experiment are averaged over operators $O_{D}$ on subsystem $D$. The physical intuition, as well as the operational interpretation of taking this average is as follows. In classical physics, chaos refers to the sensitive dependence of the system's dynamics on the initial conditions. In particular, one can imagine preparing two identical objects, adding a small perturbation to one of them, and then letting them evolve under the same Hamiltonian. If the system is chaotic, the outcomes will be drastically different, since a small initial perturbation has an exponentially growing effect.

In quantum systems, chaos can be probed by preparing a pair of objects with macroscopic entanglement, i.e., in an EPR pair (or the thermofield double state at finite temperature). Once again, one can imagine adding a small perturbation to one of the objects, and then letting them evolve under two Hamiltonians, $H$ and $H^{*}$, forward and backward in time, respectively. Without the perturbation, the system remains in an EPR pair, since

$$
e^{-i H t} \otimes e^{i H^{*} t}|\mathrm{EPR}\rangle=|\mathrm{EPR}\rangle .
$$

However, with a perturbation, the resulting dynamics can cause the system to deviate. In the above, our perturbation is $O_{A}$ and we are interested in studying how the initial $|E P R\rangle$ entanglement changes in time. Operationally, we use the EPR projector, $\Pi_{D D^{\prime}}$, to check if the entanglement (as seen from $D D^{\prime}$ ) has been disturbed by the perturbation or not. This now provides a natural bridge to explain the averaging over operators $O_{D}$ seen in Eq. (32), since $\int d O_{D} O_{D} \otimes O_{D}^{*}=\Pi_{D D^{\prime}}$.

In addition to verifying entanglement, the projector $\Pi_{D D^{\prime}}$ also has the intriguing effect of "undoing" the chaotic dynamics. To see this, let us assume that the time-evolution operator $U$ is strongly scrambling so that $\langle\overline{\mathrm{OTOC}}\rangle$ is close to its theoretical minimum, $\sim\left(1 / d_{A}^{2}\right)$. By postselecting on $|\mathrm{EPR}\rangle_{D D^{\prime}}$, one obtains an output state $\left|\Psi_{\text {out }}\right\rangle$ [Eq. (20)]. Since $\left\langle\Psi_{\text {in }}\left|\Pi_{R R^{\prime}} \Pi_{C C^{\prime}} \Pi_{D D^{\prime}}\right| \Psi_{\text {in }}\right\rangle=$ $\left(1 / d_{A}^{2}\right)$, one has

$$
\left\langle\Psi_{\text {out }}\left|\Pi_{R R^{\prime}} \Pi_{C C^{\prime}} \Pi_{D D^{\prime}}\right| \Psi_{\text {out }}\right\rangle=\frac{1}{d_{A}^{2}\langle\overline{\mathrm{OTOC}}\rangle} \approx 1
$$

Thus, the projector $\Pi_{D D^{\prime}}$ not only distills an EPR pair on $R R^{\prime}$, but also undoes the chaotic time evolution associated with $U$, returning the entire system to a set of EPR pairs. In particular, if one prepares a quantum state $|\psi\rangle$ on $A$, then the output state will be close to $|\mathrm{EPR}\rangle_{C C^{\prime}}|\mathrm{EPR}\rangle_{D D^{\prime}}|\psi\rangle_{R^{\prime}}$

The fact that the projector $\Pi_{D D^{\prime}}$ can halt the chaotic dynamics of $U$ is consistent with the traversable wormhole interpretation of the Hayden-Preskill thought experiment $[5,11,29]$. Indeed, it has been found that the growth of the wormhole interior can be stopped or slowed down by applying certain interactions, and here, $\Pi_{D D^{\prime}}$ plays the role of resetting the growth of the wormhole. Most importantly, this observation provides an additional verification method for our teleportation-based decoding protocol. Once one measures an EPR pair on $D D^{\prime}$, it is very likely that one will measure EPR pairs on other pairs of qubits if the experimental procedures are perfect and there is no decoherence.

\section{TELEPORTATION-BASED DECODING PROTOCOL: ARBITRARY NOISE AND DECOHERENCE}

In the previous section, we saw that in the absence of decoherence both $P_{\text {EPR }}$ and $F_{\text {EPR }}$ provide the same information, namely, the value of the averaged OTOC, which in the ideal case precisely captures the scrambling behavior of the unitary. We now turn to our pièce de résistance, an analysis of the decoding protocol in the presence of arbitrary noise and imperfections, as characterized via a generic quantum channel $\mathcal{Q}$. The intuition behind the protocol's ability to distinguish between scrambling and decoherence is the redundancy provided by the pair of measurements, $P_{\mathrm{EPR}}$ and $F_{\mathrm{EPR}}$, in inferring the scrambling behavior of the unitary.

The protocol proceeds in exactly the same fashion as in the previous section, except that $\mathcal{Q}$ and $\mathcal{Q}^{*}$ are now applied (rather than $U$ and $U^{*}$ ). A straightforward graphical calculation then yields the probability $P_{\mathrm{EPR}}$ associated with $\Pi_{D D^{\prime}}$ as

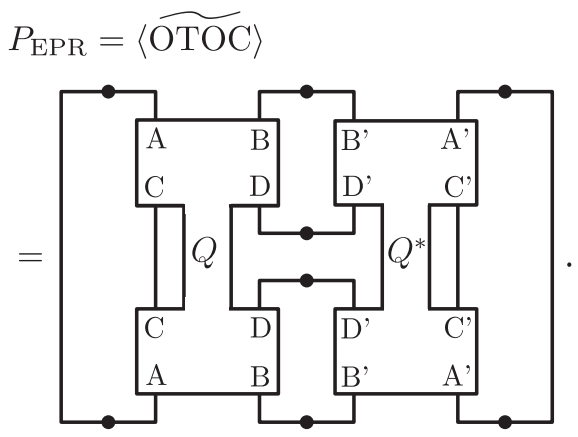

As one might recall [Eq. (13)], $\langle\widetilde{\mathrm{OTOC}}\rangle$ is directly related to the values of the Rényi-2 entropies, $S_{B^{\prime} D}^{(2)}+S_{D}^{(2)}-S_{B^{\prime}}^{(2)}$, meaning that it contains effects from both decoherence and scrambling.

To measure the mutual information, $I^{(2)}\left(R, B^{\prime} D\right)$, which encodes the true scrambling behavior of the channel $\mathcal{Q}$, we return to our previous equation for $P_{\mathrm{EPR}} F_{\mathrm{EPR}}$, wherein one finds 


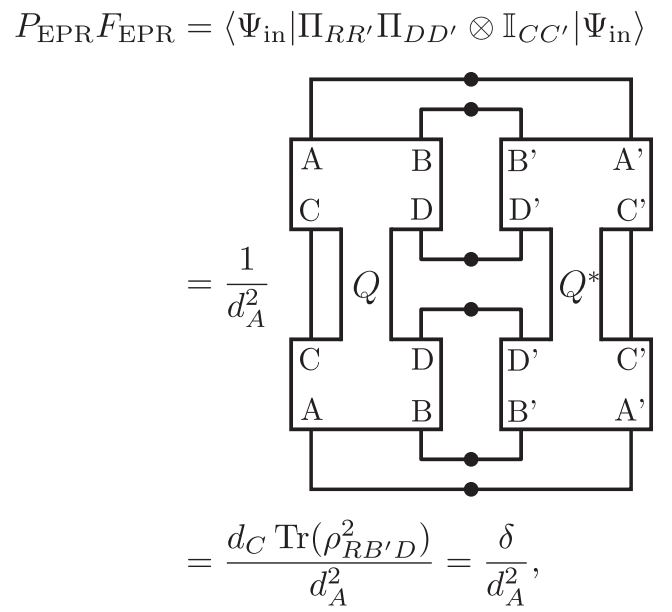

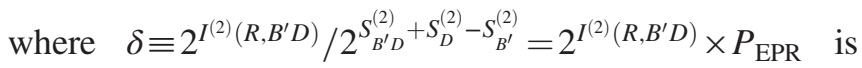
precisely our previously defined noise parameter [Eq. (15)]. We emphasize that Eqs. (36) and (37) are precisely the "noisy-quantum-channel" analogs of Eqs. (21) and (24) for the noise-free case. The decoding fidelity after postselection is then given by

$$
F_{\mathrm{EPR}}=\frac{2^{I^{(2)}\left(R, B^{\prime} D\right)}}{d_{A}^{2}} .
$$

Thus, the success of teleportation implies true scrambling [i.e., large $I^{(2)}\left(R, B^{\prime} D\right)$ ] for a generic quantum channel $\mathcal{Q}$. Moreover, by measuring both $P_{\mathrm{EPR}}$ and $F_{\mathrm{EPR}}$, one can directly compute $\delta$, thereby characterizing the amount of noise in the quantum channel.

To see this in action, let us now return to the case where $\mathcal{Q}$ reflects a depolarizing channel [Eq. (7)]. In this situation, the measurement of $\delta$ via $P_{\mathrm{EPR}}$ and $F_{\mathrm{EPR}}$ immediately provides insight into the amount of dissipation in the system (given by probability $p$ ), since

$$
\delta=\left[(1-p)^{2}+\left(2 p-p^{2}\right) \frac{1}{d_{D}^{2}}\right] .
$$

While experimental decoherence cannot always be recast simply as depolarization, this expression serves as an operational (and quantitative) measure of extrinsic experimental noise.

For the case of state decoding, an analogous calculation reveals that the error parameter $\delta$ is given by

$$
\int d \psi P_{\psi} F_{\psi}=\frac{1}{d_{A}+1}\left(P_{\mathrm{EPR}}+\frac{\delta}{d_{A}}\right) .
$$

Interestingly, we note that as an alternative strategy, one can also study the effect of decoherence for a specific input state by observing possible violations of the bound in Eq. (31).

\section{TELEPORTATION-BASED DECODING PROTOCOL: COHERENT ERRORS}

\section{A. Distinguishing scrambling from coherent errors}

In the previous section, we focused on the case of a generic noisy quantum channel and, more specifically, on the effects of depolarization. In this subsection, motivated by recent experiments [36,37], we consider the case of coherent unitary errors (i.e., systematic over- or underrotations), which lead to imperfect "backwards" time evolution (but no nonunitary decoherence). In particular, we investigate the situation where the time-evolution operator is given by $U \otimes V^{*}$ (rather than $U \otimes U^{*}$, which we assume to be the desired ideal case). For simplicity, let us assume that all other operations, including the initial preparation of EPR pairs and the final readout measurements, are error free [58].

In this scenario, the probability of measuring $|\mathrm{EPR}\rangle_{D D^{\prime}}$ is given by

$$
P_{\mathrm{EPR}}=\iint d O_{A} d O_{D}\left\langle O_{A} O_{D}(t) O_{A}^{\dagger} O_{D_{V}}^{\dagger}(t)\right\rangle=
$$

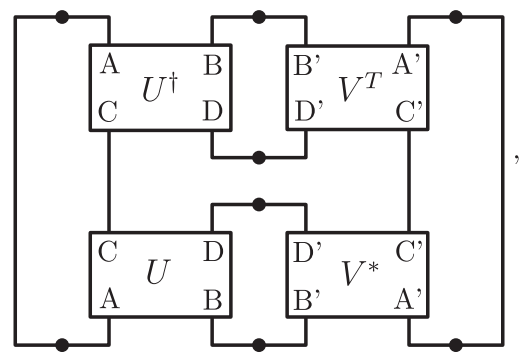

where $O_{D}(t)=U O_{D} U^{\dagger}$ and $O_{D_{V}}(t)=V O_{D} V^{\dagger}$ are time evolved by different unitaries, $U$ and $V$, respectively. A simple graphical calculation yields the product $P_{\text {EPR }} F_{\text {EPR }}$ as

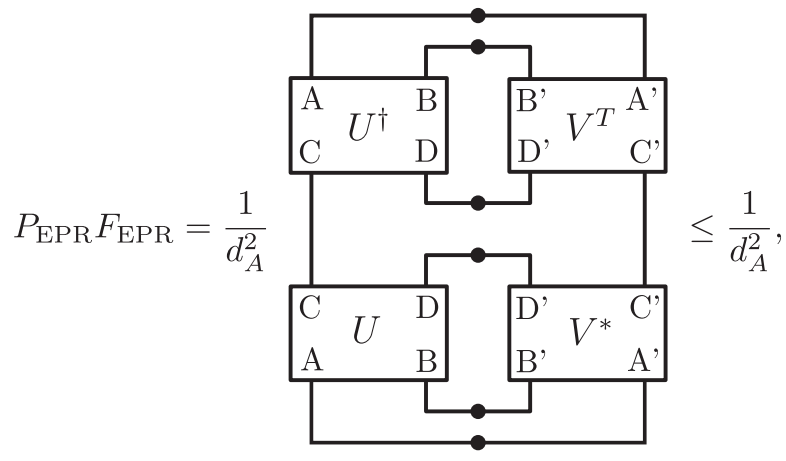

which is strictly smaller than the ideal case (i.e., when $U=V)$, where $P_{\mathrm{EPR}} F_{\mathrm{EPR}}=\left(1 / d_{A}^{2}\right)$. Again, we emphasize that Eqs. (41) and (42) are precisely the "coherent-error" analogs of Eqs. (21) and (24) in the ideal case. 
By analogy to Eq. (37), this suggests that one can define a noise parameter $\eta$ for coherent errors as follows:

$$
P_{\mathrm{EPR}} F_{\mathrm{EPR}}=\frac{\eta}{d_{A}^{2}} .
$$

While $\eta$ and $\delta$ effectively measure the same diagram, $\eta$ cannot be interpreted in terms of entropy since $U \otimes U^{*}$ is performed incorrectly.

Moreover, the physical interpretation of $\eta$ is quite different from that of $\delta$, which characterizes the strength of decoherence. In particular, we note that a natural measure of the amount of coherent error is provided by the composite unitary operator, $E=U^{\dagger} V$. In the error-free, ideal case, $E$ simply corresponds to the identity operation. The noise parameter $\eta$ is related to $E$ as follows:

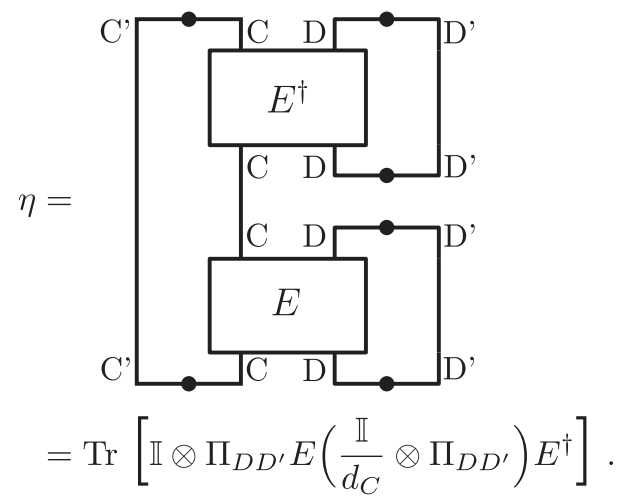

The right-hand side of Eq. (44) is the 2-norm overlap between $\left.E\right|_{D}$ and $\mathbb{I}_{D}$; here, $\left.E\right|_{D}$ is the quantum channel defined on $D$ by tracing out the degrees of freedom on $C$. If one takes $D$ to be the whole system, then $\eta$ is simply the 2-norm overlap between $E$ and $\mathbb{I}$, precisely capturing the amount of deviation between the composite unitary $U^{\dagger} V$ and the identity. Finally, we note that $0 \leq \eta \leq 1$, where the lower bound is saturated when $E=\mathbb{I} \otimes O_{D}$ for any traceless operator $O_{D}$. This contrasts with the decoherence noise parameter $\delta$, which is lower bounded by $\min \left[\left(1 / d_{A}^{2}\right),\left(1 / d_{D}^{2}\right)\right]$.

\section{B. Bounding the OTOC via $\eta$ in the case of coherent errors}

Intriguingly, under certain physical assumptions, one can utilize the measured value of $\eta$ to upper bound the true value of the OTOC that would have been measured without coherent errors (i.e., if $U=V$ ). In particular, we compare the following two quantities,

$$
\left\langle O_{A} O_{D}(t) O_{A}^{\dagger} O_{D}^{\dagger}(t)\right\rangle \text { and }\left\langle O_{A} O_{D}(t) O_{A}^{\dagger} O_{D_{V}}^{\dagger}(t)\right\rangle \text {, }
$$

and use the second, which is measured via $P_{\mathrm{EPR}}$, to bound the first. For simplicity, let us assume that $O_{A}, O_{D}$ are Pauli operators.
We also make and justify a second assumption. In particular, consider an OTOC of the form, $\left\langle O_{A}(0) O_{D}(t) O_{A}^{\dagger} O_{D}^{\prime \dagger}(t)\right\rangle$, where the two operators, $O_{D}$ and $O_{D}^{\prime}$, are both time evolved by $U$. We assume that

$$
\left\langle O_{A}(0) O_{D}(t) O_{A}^{\dagger} O_{D}^{\prime \dagger}(t)\right\rangle \approx 0,
$$

so long as $\operatorname{Tr}\left(O_{D} O_{D}^{\prime \dagger}\right)=0$. The intuition behind this assumption is as follows. At $t=0$, if regions $A$ and $D$ do not overlap, then $\left\langle O_{A}(0) O_{D}(t) O_{A}^{\dagger} O_{D}^{\prime \dagger}(t)\right\rangle=$ $\operatorname{Tr}\left(O_{D} O_{D}^{\prime \dagger}\right)=0$. Then, since OTOCs generically decay under ergodic time evolution, one expects the above expectation value to be small for all times beyond the local relaxation timescale.

To proceed, it will be useful to define a new unevolved (e.g., time $t=0$ ) operator $O_{E}=E O_{D} E^{\dagger}$, which corresponds to the conjugation of $O_{D}$ by the composite unitary $E$. The subsequent time evolution of this operator via the unitary $U$ is given by $U E O_{D} E^{\dagger} U^{\dagger}=V O_{D} V^{\dagger}=O_{D_{V}}(t)$. Then, we have

$$
\left\langle O_{A} O_{D}(t) O_{A}^{\dagger} O_{D_{V}}^{\dagger}(t)\right\rangle=\left\langle O_{A} O_{D}(t) O_{A}^{\dagger} O_{E}^{\dagger}(t)\right\rangle,
$$

where $O_{E}(t)=U O_{E} U^{\dagger}$. Let us now expand the composite unitary $E$ in terms of Pauli operators, $P$ and $Q$,

$$
E=\sum_{P, Q} \alpha_{P, Q} P \otimes Q
$$

where $P, Q$ act on subsystems $C, D$, respectively and $\sum_{P, Q}\left|\alpha_{P, Q}\right|^{2}=1$ [59]. Plugging this into our expression for $\eta$, one obtains

$$
\eta=\sum_{P}\left|\alpha_{P, \mathbb{I}}\right|^{2}
$$

Let us also expand $O_{E}$ in terms of Pauli operators,

$$
O_{E}=\sum_{P, Q} \beta_{P, Q} P \otimes Q
$$

where again $\sum_{P, Q}\left|\beta_{P, Q}\right|^{2}=1$. Plugging this expression back into Eq. (47) yields

$$
\begin{aligned}
\left\langle O_{A} O_{D}(t) O_{A}^{\dagger} O_{E}^{\dagger}(t)\right\rangle & =\sum_{P, Q} \beta_{P, Q}\left\langle O_{A} O_{D}(t) O_{A}^{\dagger}(P \otimes Q)(t)\right\rangle \\
& \approx \beta_{\mathbb{I}, O_{D}}\left\langle O_{A} O_{D}(t) O_{A}^{\dagger} O_{D}^{\dagger}(t)\right\rangle
\end{aligned}
$$

where we have used our assumption [Eq. (46)] to drop all terms with $Q \neq O_{D}$ in going from the first to the second line.

Noting that $\beta_{\mathbb{I}, O_{D}}=(1 / d) \operatorname{Tr}\left(O_{D} O_{E}^{\dagger}\right)$ allows us to bound it as follows: 


$$
\begin{aligned}
\beta_{\mathbb{I}, O_{D}} & =\frac{1}{d} \operatorname{Tr}\left[\left(\mathbb{I} \otimes O_{D}\right) E\left(\mathbb{I} \otimes O_{D}^{\dagger}\right) E^{\dagger}\right] \\
& =\frac{1}{d} \operatorname{Tr}\left[\sum_{P, Q}\left|\alpha_{P, Q}\right|^{2}\left(\mathbb{I} \otimes O_{D}\right)(P \otimes Q)\left(\mathbb{I} \otimes O_{D}^{\dagger}\right)(P \otimes Q)\right] \\
& =\sum_{P}\left|\alpha_{P, \mathbb{I}}\right|^{2}+\sum_{P} \sum_{Q \neq \mathbb{I}} \pm\left|\alpha_{P, Q}\right|^{2} \geq 2 \eta-1
\end{aligned}
$$

Here, the \pm signs in the final line correspond to the case where $O_{D}$ and $Q$ commute or anticommute, respectively. Thus, the lower bound corresponds to the case where all nonzero $\alpha_{P, Q}$ come with a negative sign.

Finally, combining Eqs. (47), (51), and (52) yields the following bound:

$$
\begin{aligned}
\left\langle O_{A} O_{D}(t) O_{A}^{\dagger} O_{D_{V}}^{\dagger}(t)\right\rangle & =\left\langle O_{A} O_{D}(t) O_{A}^{\dagger} O_{E}^{\dagger}(t)\right\rangle \\
& =\beta_{\mathbb{I}, O_{D}}\left\langle O_{A} O_{D}(t) O_{A}^{\dagger} O_{D}^{\dagger}(t)\right\rangle \\
& \geq(2 \eta-1)\left\langle O_{A} O_{D}(t) O_{A}^{\dagger} O_{D}^{\dagger}(t)\right\rangle .
\end{aligned}
$$

Thus, in the case of coherent errors corresponding to imperfect backwards time evolution, the experimentally measured value of the averaged OTOC (via, e.g., $P_{\mathrm{EPR}}$ ) explicitly bounds the actual ideal OTOC:

$$
\langle\overline{\mathrm{OTOC}}\rangle \leq \frac{P_{\mathrm{EPR}}}{2 \eta-1} .
$$

We note that this bound is valid only for $\eta>0.5$.

Two additional remarks. First, it is worth pointing out that the value of $\beta_{\mathbb{I}, O_{D}}$ can be directly measured via $\left\langle O_{A} O_{D}(t) O_{A}^{\dagger} O_{D_{V}}^{\dagger}(t)\right\rangle$ with $O_{A}=\mathbb{I}$, since $\beta_{\mathbb{I}, O_{D}}=$ $\left\langle O_{D} O_{E}^{\dagger}(t)\right\rangle=\left\langle O_{D}(t) O_{D_{V}}^{\dagger}(t)\right\rangle$. Second, in a generic chaotic system, one expects the \pm signs in Eq. (52) to appear randomly. Under this assumption, one can make the following approximation:

$$
\beta_{\mathbb{I}, O_{D}} \approx \sum_{P}\left|\alpha_{P, \mathbb{I}}\right|^{2}=\eta
$$

which enables us to obtain an estimate for the actual value of the OTOC and not simply a bound:

$$
\left\langle O_{A} O_{D}(t) O_{A}^{\dagger} O_{D}^{\dagger}(t)\right\rangle \approx \frac{1}{\eta}\left\langle O_{A} O_{D}(t) O_{A}^{\dagger} O_{D_{V}}^{\dagger}(t)\right\rangle
$$

\section{BOUNDING THE MUTUAL INFORMATION VIA THE DECODING FIDELITY}

\section{A. Mutual information bound}

In the previous section, we have shown that in the case of coherent errors, one can utilize $\eta$ as extracted from $P_{\text {EPR }}$ and $F_{\mathrm{EPR}}$ to formally bound the true (i.e., error-free) value of the averaged OTOC. However, this proof explicitly hinges on the unitarity of the composite channel $E$ and is thus inapplicable to the generic situation with decoherence. Moreover, in the presence of decoherence, it becomes ambiguous to define what precisely the value of the OTOC is [60]; rather, as we have previously seen, a better characterization for quantum scrambling is provided by the mutual information.

To this end, in this section, we demonstrate that for arbitrary quantum channels, one can derive a bound on the mutual information, $I^{(2)}\left(R, B^{\prime} D\right)$, using only the decoding fidelity $F_{\mathrm{EPR}}$ [61]. When applied to the case of purely unitary errors (i.e., the previous section), this leads to a somewhat weaker bound on $\langle\overline{\mathrm{OTOC}}\rangle$.

To treat experimental imperfections on a fully general footing, we consider time evolution via the quantum channel $\mathcal{Q}$ and an arbitrary decoding operation $\Phi$, acting nontrivially only on $B^{\prime} D$ (Fig. 3). As previously discussed, the goal of this decoding operation is to distill an EPR pair on $R \bar{R}$, where $\bar{R}$ represents a subset of the qubits in $B^{\prime} D$ with the same dimension as $R$ (e.g., $|R|=|\bar{R}|$ ). Let us assume that $\Phi$, an arbitrary completely positive tracepreserving map, outputs a normalized state supported on $R \bar{R}$ :

$$
\Phi: \rho_{R B^{\prime} D} \rightarrow \sigma_{R \bar{R}}
$$

Since the decoding operation acts locally on $B^{\prime} D$, it cannot increase entanglement between $R$ and $B^{\prime} D$; i.e., the mutual information satisfies $I\left(R, B^{\prime} D\right) \geq I(R, \bar{R})$ [62]. Since $I(R, \bar{R})$ can be lower bounded via $F_{\mathrm{EPR}}$, any nontrivial decoding fidelity always signifies quantum scrambling even in the presence of arbitrary imperfections.

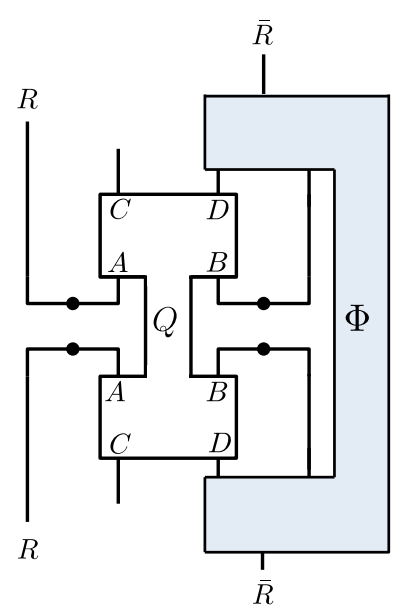

FIG. 3. Schematic representation of the decoding protocol as the distillation of an EPR pair on $R \bar{R}$. The initial state is $|\mathrm{EPR}\rangle_{R A}|\mathrm{EPR}\rangle_{B B^{\prime}}$. The quantum channel $\mathcal{Q}$ describes the time evolution of the system and $\Phi$ represents an arbitrary decoding operation acting only on $B^{\prime} D$. 
Deriving a rigorous lower bound on either the von Neumann or Rényi-2 mutual informations in terms of $F_{\text {EPR }}$ is tremendously challenging, owing in part to the existence of fine-tuned adversarial examples that tend to make the bounds loose in physically relevant situations. This is ameliorated by making the assumption that the decoding fidelity is independent of the input state $|\psi\rangle$. One expects this assumption to be approximately valid for strongly interacting systems without conserved quantities after the system locally thermalizes. This assumption also excludes the trivial decoding protocol which returns a fixed state regardless of input state.

To begin, we note that $\rho_{R}$ is a maximally mixed state [Eq. (11)], implying that $\sigma_{R}$ is also maximally mixed, since $\Phi$ acts only locally on $B^{\prime} D$. Moreover, our assumption that the decoding fidelity does not depend on the input state implies that $\sigma_{\bar{R}}$ is a maximally mixed state as well. While generally true, this statement is particularly easy to see in the case where $R$ and $\bar{R}$ consist of only single qubits. In particular, we can use our previous trick and decompose $\sigma_{R \bar{R}}$ in terms of Pauli operators: $\sigma_{R \bar{R}}=\sum_{P, Q} \gamma_{P, Q} P \otimes Q$, for $P, Q \in\{\mathbb{I}, X, Y, Z\}$. Since $\sigma_{R}$ is maximally mixed, one has that $\gamma_{P, \mathbb{I}}=0$ for all $P \neq \mathbb{I}$.

For an input state $|\psi\rangle$, the quantum state on $\bar{R}$ is given by

$$
d_{R}\left(|\psi\rangle\langle\psi| \otimes \mathbb{I}_{\bar{R}}\right) \sigma_{R \bar{R}}\left(|\psi\rangle\langle\psi| \otimes \mathbb{I}_{\bar{R}}\right),
$$

where $d_{R}$ is a normalization constant. The decoding fidelity can then be written as

$$
F_{\psi}=d_{R} \operatorname{Tr}\left(|\psi\rangle\left\langle\psi|\otimes| \psi^{*}\right\rangle\left\langle\psi^{*}\right| \sigma_{R \bar{R}}\right) .
$$

Noting that $|0\rangle\langle 0|=(\mathbb{I}+Z) / 2$ and $|1\rangle\langle 1|=(\mathbb{I}-Z) / 2$ implies $\gamma_{Z, \mathbb{I}}+\gamma_{\mathbb{I}, Z}=0$; since $\gamma_{Z, \mathbb{I}}=0$, one also has that $\gamma_{\mathbb{I}, Z}=0$. The same analysis leads to $\gamma_{\mathbb{I}, P}=0$ for all nonidentity Pauli operators $P$. Thus, $\sigma_{\bar{R}}$ is also a maximally mixed state.

Having shown that $\sigma_{\bar{R}}$ is a maximally mixed state, we are now ready to lower bound the mutual information. The EPR projector and the decoding fidelity of the distilled quantum state $\sigma_{R \bar{R}}$ are given by $\Pi_{R \bar{R}}=|\mathrm{EPR}\rangle\left\langle\left.\mathrm{EPR}\right|_{R \bar{R}}\right.$ and $F_{\mathrm{EPR}}=\operatorname{Tr}\left(\Pi_{R \bar{R}} \rho_{R \bar{R}}\right)$, respectively. Then, using the Cauchy-Schwartz inequality, one immediately arrives at the following bound:

$$
\begin{aligned}
S_{R \bar{R}}^{(2)} & =-\log _{2} \operatorname{Tr}\left(\rho_{R \bar{R}}^{2}\right) \leq-\log _{2} \operatorname{Tr}\left(\Pi_{R \bar{R}} \rho_{R \bar{R}}\right) \operatorname{Tr}\left(\Pi_{R \bar{R}} \rho_{R \bar{R}}\right) \\
& =-2 \log _{2} F_{\mathrm{EPR}},
\end{aligned}
$$

implying that the mutual information satisfies

$I^{(2)}(R, \bar{R})=S_{R}+S_{\bar{R}}-S_{R \bar{R}} \geq 2 \log _{2} d_{R}+2 \log _{2} F_{\mathrm{EPR}}$.

In order to utilize the monotonicity of mutual information [53], we will make the additional technical assumption that the Rényi-2 and von Neumann entropies are close to one another [63]. This then leads to our final result, lower bounding the mutual information in terms of the decoding fidelity:

$I\left(R, B^{\prime} D\right) \approx I^{(2)}\left(R, B^{\prime} D\right) \geq 2 \log _{2} d_{R}+2 \log _{2} F_{\mathrm{EPR}}$.

\section{B. OTOC bound for coherent errors}

While the previous subsection focused on the case of arbitrary quantum channels, one can also apply the derived bound to the situation where only coherent errors are present. To this end, let us return to the scenario described in Sec. VI. A, where the time evolution is given by $U \otimes V^{*}$. As we have already seen, the measurement of $P_{\mathrm{EPR}}$ corresponds to

$$
P_{\mathrm{EPR}}=\int d O_{A} d O_{D}\left\langle O_{A} O_{D}(t) O_{A}^{\dagger} O_{D_{V}}^{\dagger}(t)\right\rangle,
$$

which includes the effect of unitary errors associated with $E=U^{\dagger} V \neq \mathbb{I}$. In analogy to Sec. VI B, the true OTOC, which would have been measured if the experiment did not contain such unitary errors, is given by

$$
\overline{\langle\mathrm{OTOC}\rangle}=\int d O_{A} d O_{D}\left\langle O_{A} O_{D}(t) O_{A}^{\dagger} O_{D}^{\dagger}(t)\right\rangle .
$$

Since $\overline{\langle\mathrm{OTOC}\rangle}=2^{-I^{(2)}(A, B D)}$, our above bound on the mutual information also immediately bounds $\overline{\langle\mathrm{OTOC}\rangle}$ in the case of purely coherent errors:

$$
\overline{\langle\mathrm{OTOC}\rangle} \leq \frac{1}{d_{R}^{2} F_{\mathrm{EPR}}^{2}} .
$$

\section{EXPERIMENTAL IMPLEMENTATION}

Having detailed a teleportation protocol that explicitly enables experiments to distinguish between decoherence and quantum information scrambling [64], we now propose two specific examples of scrambling Clifford circuits [49] amenable to near-term experiments in small-scale quantum simulators $[67,68]$.

\section{A. Qubit Clifford scrambler}

Let us consider the following 3-qubit unitary operator:

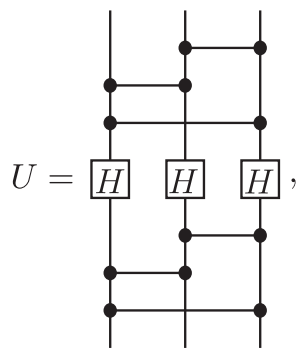




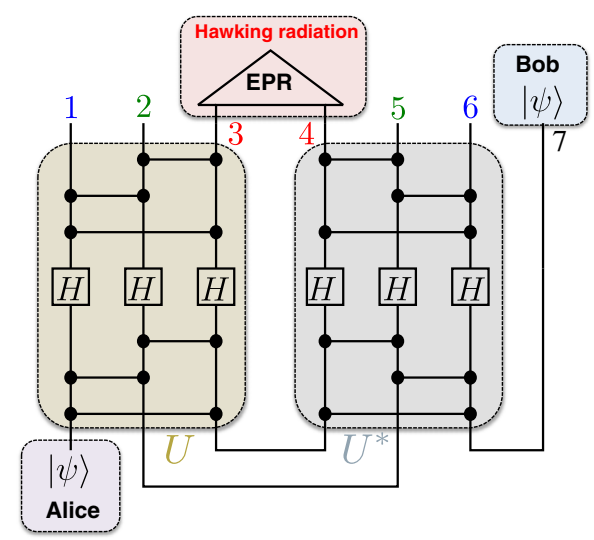

FIG. 4. Decoding circuit based upon a 3-qubit Clifford scrambler. Alice's quantum state $|\psi\rangle$ is supported on qubit 1 , while Bob's quantum register corresponds to qubit 7. The left (beige) and right (gray) Hilbert spaces have the following correspondences $1 \leftrightarrow 6,2 \leftrightarrow 5$, and $3 \leftrightarrow 4$ (with respect to $U \otimes U^{*}$ ). By performing an EPR projection on qubits 3 and 4, Bob teleports Alice's quantum state to his register qubit. In the case of this Clifford scrambler, Bob could also have achieved teleportation by performing EPR projections on either qubits $\{1,6\}$ or $\{2,5\}$. This distinguishes the Clifford scrambler from other more trivial (nonscrambling) unitaries (i.e., a SWAP gate), where teleportation occurs only for EPR projection on a specific pair of qubits.

where $H$ represents a Hadamard gate, while 2-qubit, control- $Z$ gates $\left(|i, j\rangle \rightarrow(-1)^{i j}|i, j\rangle\right)$ are depicted as horizontal lines (ending in dots). This unitary is maximally scrambling since all one-body Pauli operators are delocalized into three-body Pauli operators under $U$ [69]. From the perspective of decoding, this delocalization implies that Bob can collect any pair of qubits (from among the three possible pairs in Fig. 4) and perform a projective measurement in order to decode Alice's state. To be concrete, the full decoding protocol is illustrated in Fig. 4.

Two comments are in order. In particular, for a Haar random unitary, one expects $\langle\overline{\mathrm{OTOC}}\rangle_{S}=\frac{7}{16}$, whereas our circuit exhibits $\langle\overline{\mathrm{OTOC}}\rangle_{S}=\frac{1}{4}$. This discrepancy arises from finite-size effects, since one expects a Haar random unitary to saturate the lower bound of $1 / 4$ only in the limit of large systems, i.e., $d, d_{D} \rightarrow \infty$ while fixing $d_{R}=2$. On the other hand, our Clifford circuit saturates this lower bound by construction but has certain nongeneric features [49]. Second, as we briefly alluded to in Sec. IV C, it is also possible to explore circuits that scramble only classical information:

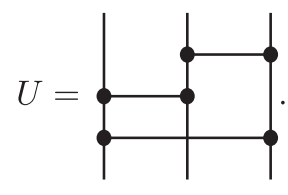

In this case, teleportation occurs only for computational basis states.

\section{B. Qutrit Clifford scrambler}

While we presented the minimum case of interest for qubits in the previous subsection, by increasing the on-site Hilbert space, one can realize slightly more complex circuits in even smaller systems. To this end, motivated by the advent of physical qutrit implementations ranging from solid-state spin defects and superconducting circuits to orbital angular momentum states of photons, we describe a simple qutrit Clifford scrambler.

To begin, we denote a qutrit as a three-state quantum spin with basis $|0\rangle,|1\rangle,|2\rangle$. An elementary entangling gate between two qutrits can be achieved via the following controlled-NOT gate:

$$
\mathrm{CNOT}_{1 \rightarrow 2}|i, j\rangle=|i, i+j\rangle \quad \text { modulo 3, }
$$

where the subscript $1 \rightarrow 2$ indicates that the control is qutrit- 1 and the target is qutrit-2. Switching the control and target realizes an analogous operation: $\mathrm{CNOT}_{2 \rightarrow 1}|i, j\rangle=$ $|i+j, i\rangle \bmod 3$.

Let us now consider the following qutrit unitary:

$$
U=\mathrm{CNOT}_{2 \rightarrow 1} \mathrm{CNOT}_{1 \rightarrow 2},
$$

which can be explicitly decomposed as $U|i, j\rangle=|2 i+j, i+j\rangle$ or graphically reexpressed as

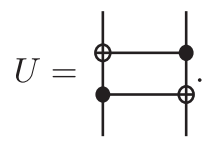

To understand the scrambling properties of this unitary, we will explore how the qutrit Pauli operators are transformed under the circuit. In particular, let us consider the following qutrit Pauli operators: $X=\sum_{j=0}^{2}|j+1\rangle\langle j|$ and $Z=\sum_{j=0}^{2} \omega^{j}|j\rangle\langle j|$, where $\omega=e^{i(2 \pi / 3)}$.

One finds that these operators are transformed as follows:

$$
\begin{aligned}
& U(Z \otimes I) U^{\dagger}=Z \otimes Z^{2}, \\
& U(I \otimes Z) U^{\dagger}=Z^{2} \otimes Z^{2}, \\
& U(X \otimes I) U^{\dagger}=X^{2} \otimes X, \\
& U(I \otimes X) U^{\dagger}=X \otimes X .
\end{aligned}
$$

Thus, as in the qubit case, we observe that the unitary transforms any nonidentity one-body Pauli operator into a two-body operator. This property is essential for the delocalization of quantum information and enables the construction of a similar decoding protocol: 


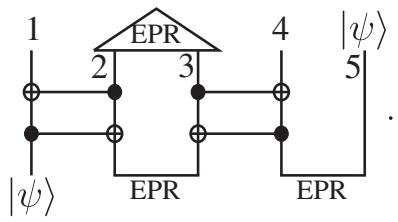

By performing an EPR projection on either qutrits $\{2,3\}$ or $\{1,4\}$, Bob successfully teleports Alice's quantum state from qutrit-1 to qutrit-5.

\section{Distinction from conventional quantum teleportation}

The importance of being able to perform teleportation by projecting either pair of qutrits (or in the previous case, any of the three qubit pairs) is most easily seen by considering the effect of a SWAP gate, $\operatorname{SWAP}|i, j\rangle=|j, i\rangle$, or graphically,

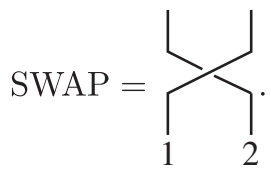

From the perspective of scrambling, a SWAP gate is totally trivial since it does not generate any entanglement; thus, its decoding behavior must be markedly different from that of the maximally scrambling $U$ in Eq. (70).

Replacing $U$ with the SWAP gate in the decoding protocol leads to the following:

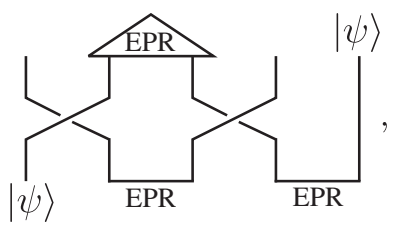

which is simply ordinary quantum teleportation $[53,70,71]$. Crucially, this teleportation only works when Bob projects on qutrits $\{2,3\}$ and fails if he attempts to project on qutrits $\{1,4\}$. Herein lies the essential feature of a maximally scrambling unitary: Successful decoding and teleportation occur regardless of which pair of qutrits (or qubits) one chooses to collect and project, precisely indicating the full delocalization of quantum information across the circuit.

\section{CONCLUSION}

We demonstrate that one can distinguish between scrambling and decoherence in strongly interacting quantum systems by utilizing a teleportation-based decoding protocol. Our protocol enables the explicit extraction of a "noise parameter," which can be used to bound the value of out-oftime-ordered correlation functions in the case of coherent errors. More generally, even for arbitrary imperfections, the teleportation fidelity acts as a metric for quantum scrambling and enables the bounding of the mutual information between subsystems. Thus, our protocol represents the first example of an experimental method, which can unambiguously characterize the delocalization of quantum information within a system's own degrees of freedom and differentiate this from entanglement with an extrinsic environment.

Our work opens the door to a number of intriguing future directions. First, by systematically exploring the statedependent decoding fidelity in the presence of different forms of decoherence, one may be able to study the transition from classical to quantum chaos. Second, in this work, we have mainly focused on decoherence as an adversary to quantum scrambling. However, the pronounced sensitivity of scrambling dynamics to the presence of decoherence suggests that one may be able to utilize our protocol as a particularly efficient "noise" spectroscopy tool [72]. Finally, an interesting question that has received much recent attention, and which goes under the moniker of quantum supremacy, is whether quantum devices without error correction can perform computational tasks beyond the capabilities of classical computers [73]. It has been suggested that the simulation of random quantum circuits may be an ideal platform for this purpose [74]. Since OTOCs are natural probes of pseudorandomness, it may be possible to generalize our protocol to explore such questions.

\section{ACKNOWLEDGMENTS}

We gratefully acknowledge the insights of and discussions with P. Hess, A. Kyprianidis, G. Pagano, J. Zhang, V. Ramasesh, M. Blok, I. Siddiqi, C. Monroe, and Q. Zhuang. We are particularly indebted to T. Schuster for a careful reading of the manuscript and many helpful discussions. This work was supported by the Office of Science, Office of High Energy Physics, of the U.S. Department of Energy under Contract No. DE-AC0205CH11231 through the COMPHEP pilot, "Probing information scrambling via quantum teleportation," and the Office of Advanced Scientific Computing Research, Quantum Algorithm Teams Program.

Note added.-Recently, an experimental implementation of our protocol has been realized in a trapped ion quantum simulator [75], enabling the experiment to explicitly differentiate between the effects of scrambling and decoherence.

[1] J. M. Deutsch, Quantum Statistical Mechanics in a Closed System, Phys. Rev. A 43, 2046 (1991).

[2] M. Srednicki, Chaos and Quantum Thermalization, Phys. Rev. E 50, 888 (1994). 
[3] H. Tasaki, From Quantum Dynamics to the Canonical Distribution: General Picture and a Rigorous Example, Phys. Rev. Lett. 80, 1373 (1998).

[4] M. Rigol, V. Dunjko, and M. Olshanii, Thermalization and Its Mechanism for Generic Isolated Quantum Systems, Nature (London) 452, 854 (2008).

[5] P Hayden and J. Preskill, Black Holes as Mirrors: Quantum Information in Random Subsystems, J. High Energy Phys. 09 (2007) 120.

[6] Y. Sekino and L. Susskind, Fast Scramblers, J. High Energy Phys. 10 (2008) 065.

[7] N. Lashkari, D. Stanford, M. Hastings, T. Osborne, and P. Hayden, Towards the Fast Scrambling Conjecture, J. High Energy Phys. 04 (2013) 022.

[8] A. Kitaev, A Simple Model of Quantum Hologarphy (part 1), http://online.kitp.ucsb.edu/online/entangled15/kitaev/; $A$ Simple Model of Quantum Hologarphy (part 2), http:// online.kitp.ucsb.edu/online/entangled15/kitaev2/.

[9] J. Maldacena and D. Stanford, Remarks on the Sachdev-YeKitaev Model, Phys. Rev. D 94, 106002 (2016).

[10] S. H. Shenker and D. Stanford, Black Holes and the Butterfly Effect, J. High Energy Phys. 03 (2014) 067.

[11] J. Maldacena, D. Stanford, and Z. Yang, Diving into Traversable Wormholes, Fortschr. Phys. 65, 1700034 (2017).

[12] D. A. Roberts, D. Stanford, and L. Susskind, Localized Shocks, J. High Energy Phys. 03 (2015) 051.

[13] S. H. Shenker and D. Stanford, Multiple Shocks, J. High Energy Phys. 12 (2014) 046.

[14] D. A. Roberts and D. Stanford, Two-Dimensional Conformal Field Theory and the Butterfly Effect, Phys. Rev. Lett. 115, 131603 (2015).

[15] P. Hosur, X.-L. Qi, D. A. Roberts, and B. Yoshida, Chaos in Quantum Channels, J. High Energy Phys. 02 (2016) 004.

[16] D. A. Roberts and B. Yoshida, Chaos and Complexity by Design, J. High Energy Phys. 04 (2017) 121.

[17] M. Blake, Universal Charge Diffusion and the Butterfly Effect, Phys. Rev. Lett. 117, 091601 (2016).

[18] M. Blake, Universal Diffusion in Incoherent Black Holes, Phys. Rev. D 94, 086014 (2016).

[19] A. Kitaev, Hidden Correlations in the Hawking Radiation and Thermal Noise, http://online.kitp.ucsb.edu/online/ joint98/kitaev/.

[20] A. Nahum, J. Ruhman, S. Vijay, and J. Haah, Quantum Entanglement Growth under Random Unitary Dynamics, Phys. Rev. X 7, 031016 (2017).

[21] C. von Keyserlingk, T. Rakovszky, F. Pollmann, and S. Sondhi, Operator Hydrodynamics, OTOCs, and Entanglement Growth in Systems without Conservation Laws, Phys. Rev. X 8, 021013 (2018).

[22] J. Cotler, N. Hunter-Jones, J. Liu, and B. Yoshida, Chaos, Complexity, and Random Matrices, J. High Energy Phys. 11 (2017) 48.

[23] R. Fan, P. Zhang, H. Shen, and H. Zhai, Out-of-Time-Order Correlation for Many-Body Localization, Sci. Bull. 62, 707 (2017).

[24] V. Khemani, A. Vishwanath, and D. A. Huse, Operator Spreading and the Emergence of Dissipation in Unitary Dynamics with Conservation Laws, Phys. Rev. X 8, 031057 (2018).
[25] R. A. Davison, W. Fu, A. Georges, Y. Gu, K. Jensen, and S. Sachdev, Thermoelectric Transport in Disordered Metals without Quasiparticles: The Sachdev-YeKitaev Models and Holography, Phys. Rev. B 95, 155131 (2017).

[26] Y. Gu, X.-L. Qi, and D. Stanford, Local Criticality, Diffusion and Chaos in Generalized Sachdev-Ye-Kitaev Models, J. High Energy Phys. 05 (2017) 125.

[27] P. Gao, D. L. Jafferis, and A. Wall, Traversable Wormholes via a Double Trace Deformation, J. High Energy Phys. 12 (2017) 151.

[28] D. N. Page, Average Entropy of a Subsystem, Phys. Rev. Lett. 71, 1291 (1993).

[29] V. Khemani, A. Vishwanath, and D. A. Huse, Operator Spreading and the Emergence of Dissipative Hydrodynamics under Unitary Evolution with Conservation Laws, Phys. Rev. X 8, 031057 (2018).

[30] S. Banerjee and E. Altman, Solvable Model for a Dynamical Quantum Phase Transition from Fast to Slow Scrambling, Phys. Rev. B 95, 134302 (2017).

[31] A. A. Patel and S. Sachdev, Quantum Chaos on a Critical Fermi Surface, Proc. Natl. Acad. Sci. U.S.A. 114, 1844 (2017).

[32] A. I. Larkin and Y.N. Ovchinnikov, Quasiclassical Method in the Theory of Superconductivity, Sov. Phys. JETP 28, 1200 (1969), http://jetp.ac.ru/cgi-bin/dn/ e_028_06_1200.pdf.

[33] B. Swingle, G. Bentsen, M. Schleier-Smith, and P. Hayden, Measuring the Scrambling of Quantum Information, Phys. Rev. A 94, 040302 (2016).

[34] N. Y. Yao, F. Grusdt, B. Swingle, M. D. Lukin, D. M. Stamper-Kurn, J.E. Moore, and E. A. Demler, Interferometric Approach to Probing Fast Scrambling, arXiv: 1607.01801.

[35] G. Zhu, M. Hafezi, and T. Grover, Measurement of ManyBody Chaos Using a Quantum Clock, Phys. Rev. A 94, 062329 (2016).

[36] M. Gärttner, J. G. Bohnet, A. Safavi-Naini, M. L. Wall, J. J. Bollinger, and A. M. Rey, Measuring Out-ofTime-Order Correlations and Multiple Quantum Spectra in a Trapped-Ion Quantum Magnet, Nat. Phys. 13, 781 (2017).

[37] J. Li, R. Fan, H. Wang, B. Ye, B. Zeng, H. Zhai, X. Peng, and J. Du, Measuring Out-of-Time-Order Correlators on a Nuclear Magnetic Resonance Quantum Simulator, Phys. Rev. X 7, 031011 (2017).

[38] Z. Hradil, Quantum-State Estimation, Phys. Rev. A 55, R1561 (1997).

[39] V. V. Dodonov and V.I. Man'ko, Positive Distribution Description for Spin States, Phys. Lett. A 229, 335 (1997).

[40] H. Häffner, W. Hänsel, C. F. Roos, J. Benhelm, M. Chwalla, T. Körber, U. D. Rapol, M. Riebe, P. O. Schmidt, C. Becher et al., Scalable Multiparticle Entanglement of Trapped Ions, Nature (London) 438, 643 (2005).

[41] D. Gross, Y.-K. Liu, S. T. Flammia, S. Becker, and J. Eisert, Quantum State Tomography via Compressed Sensing, Phys. Rev. Lett. 105, 150401 (2010).

[42] B. Swingleand N. Y. Halpern, Resilience of Scrambling Measurements, Phys. Rev. A 97, 062113 (2018). 
[43] L. K. Grover, A Fast Quantum Mechanical Algorithm for Database Search, in Proceedings of the Twenty-Eighth Annual ACM Symposium on Theory of Computing (Association for Computing Machinery, New York, 1996), pp. 212-219, DOI: 10.1145/237814.237866.

[44] Y. Huang, F. G. S. L. Brandao, and Y.-L. Zhang, Finite-Size Scaling of Out-of-Time-Ordered Correlators at Late Times, arXiv:1705.07597.

[45] Equation (3) holds since the Pauli operators form a unitary 1-design $[46,47]$. Note that there are $d_{A}^{2}, d_{D}^{2}$ Pauli operators (including the identity operator) on regions $A, D$, respectively.

[46] J. M. Renes, R. Blume-Kohout, A. J. Scott, and C. M. Caves, Symmetric Informationally Complete Quantum Measurements, J. Math. Phys. 45, 2171 (2004).

[47] C. Dankert, R. Cleve, J. Emerson, and E. Livine, Exact and Approximate Unitary 2-designs and Their Application to Fidelity Estimation, Phys. Rev. A 80, 012304 (2009).

[48] We note that there are unitary operators which satisfy Eq. (4), but not Eq. (1). For example, a random Clifford operator is scrambling for Eq. (4), since the Clifford operators form a unitary 2-design. However, OTOCs for a Clifford unitary are always \pm 1 if $O_{X}=O_{Z}$ and $O_{Y}=O_{W}$ are Pauli operators, and thus do not satisfy Eq. (1). In this sense, a random unitary from a 2-design is not enough to achieve full scrambling. Rather, to achieve full scrambling, it suffices to pick a random operator $U$ from a unitary 4-design.

[49] See Supplemental Material at http://link.aps.org/ supplemental/10.1103/PhysRevX.9.011006 for additional details and derivations.

[50] P. Hayden, M. Horodecki, A. Winter, and J. Yard, A Decoupling Approach to the Quantum Capacity, Open Syst. Inf. Dyn. 15, 7 (2008).

[51] It is possible to generalize our results to finite temperature (factorable ensembles) using the Rényi divergence [49].

[52] While the Rényi-2 mutual information is a measurable quantity as the average of OTOCs, the standard mutual information $(\alpha=1)$ is often more convenient as it satisfies useful monotonicity inequalities. For the case of maximally mixed ensembles $\rho=(1 / d) \mathbb{I}$, one can derive $\mathrm{I}\left(A, B^{\prime} D\right) \geq$ $\mathrm{I}^{(2)}\left(A, B^{\prime} D\right)$ using the monotonicity of Rényi entropy. This analysis can be generalized to cases where the input and output ensembles factorize [49]; $\rho_{A B}=\rho_{A} \otimes \rho_{B}$ and $\rho_{C D}=\rho_{C} \otimes \rho_{D}$, where the Rényi-2 mutual information is replaced with a certain expression involving the Rényi-2 divergence from which the standard mutual information can be lower bounded [49].

[53] M. A. Nielsen and I. L. Chuang, Quantum Computation and Quantum Information (Cambridge University Press, Cambridge, England, 2010).

[54] We note that in the presence of arbitrary forms of noise and decoherence, the experimentally "measured" value of the OTOC may depend on the specific measurement protocol. For example, the OTOC measured via interferometric protocols [33-37] will generically differ from the OTOC measured via our teleportation protocol. However, for the important case of a purely depolarizing channel as per Eq. (7), all such protocols will measure the same OTOC given by Eq. (8). Moreover, while the quantitative values of noisy OTOCs may differ between protocols, their qualitative decay in the presence of decoherence is generic. To this end, a key difference between our decoding protocol and previously proposed interferometric protocols is the initial preparation of EPR pairs; this preparation is not present in the case of interferometric protocols and underlies the reason why our teleportation-based method can verify the existence of scrambling dynamics while prior methods cannot.

[55] We do not know the terminology for the expression $S_{B D}^{(2)}+S_{D}^{(2)}-S_{B}^{(2)}$, but it is worth noting that $S_{B D}+S_{D}-$ $S_{B} \geq 0$ corresponds to the celebrated Araki-Lieb inequality.

[56] To derive this lower bound, we again insert an EPR projector on $C C^{\prime}$ into the diagram for $P_{\psi} F_{\psi}$.

[57] This integral over $|\psi\rangle$ can be replaced with an average over a set of states that form a 2-design. One example is the set of eigenstates of the Pauli operators.

[58] Note that coherent errors in the initial EPR preparation can also be absorbed into the definition of $V$.

[59] Note that this normalization condition is implied by the unitarity of $E$.

[60] The intuition behind this ambiguity is that for a generic quantum channel, one can decompose its action using Kraus operators, but this decomposition is not unique [53].

[61] The key point here is that $F_{\text {EPR }}$ always provides a lower bound on the mutual information between $R$ and $B^{\prime} D$, regardless of the nature of experimental errors. Stated differently, in the context of the black hole information problem, the fact that one can retrieve a quantum state from the Hawking radiation (i.e., the teleportation is successful) implies that the system has scrambled, regardless of how one performs the decoding.

[62] Note that we are using the von Neumann mutual information here.

[63] Recall that Rényi-2 mutual information is not monotonically decreasing in general [49].

[64] Notable examples of fast quantum information scramblers include the Sachdev-Ye-Kitaev model [19,65], $k$-local random spin models [66], and random quantum circuits [47].

[65] S. Sachdev and J. Ye, Gapless Spin-Fluid Ground State in a Random Quantum Heisenberg Magnet, Phys. Rev. Lett. 70, 3339 (1993).

[66] L. Erdős and D. Schröder, Phase Transition in the Density of States of Quantum Spin Glasses, Math. Phys. Anal. Geom. 17, 441 (2014).

[67] J. Zhang, G. Pagano, P. W. Hess, A. Kyprianidis, P. Becker, H. Kaplan, A. V. Gorshkov, Z.-X. Gong, and C. Monroe, Observation of a Many-Body Dynamical Phase Transition with a 53-Qubit Quantum Simulator, Nature (London) 551, 601 (2017).

[68] H. Bernien, S. Schwartz, A. Keesling, H. Levine, A. Omran, H. Pichler, S. Choi, A.S. Zibrov, M. Endres, M. Greiner et al., Probing Many-Body Dynamics on a 51-Atom Quantum Simulator, Nature (London) 551, 579 (2017). 
[69] F. Pastawski, B. Yoshida, D. Harlow, and J. Preskill, Holographic Quantum Error-Correcting Codes: Toy Models for the Bulk/Boundary Correspondence, J. High Energy Phys. 06 (2015) 149.

[70] D. Bouwmeester, J.-W. Pan, K. Mattle, M. Eibl, H. Weinfurter, and A. Zeilinger, Experimental Quantum Teleportation, Nature (London) 390, 575 (1997).

[71] A. Furusawa, J. L. Sørensen, S. L. Braunstein, C. A. Fuchs, H. J. Kimble, and E.S. Polzik, Unconditional Quantum Teleportation, Science 282, 706 (1998).

[72] An interesting possibility along these lines is to investigate the higher moments of the underlying probability distribution associated with $P_{\mathrm{EPR}}$ in order to diagnose temporal correlations in the noise spectrum.

[73] J. Preskill, Quantum Computing in the NISQ Era and Beyond, Quantum 2, 79 (2018).

[74] S. Boixo, S. V. Isakov, V. N. Smelyanskiy, R. Babbush, N. Ding, Z. Jiang, J. M. Martinis, and H. Neven, Characterizing Quantum Supremacy in Near-Term Devices, Nat. Phys. 14, 595 (2018).

[75] K. A. Landsman, C. Figgatt, T. Schuster, N. M. Linke, B. Yoshida, N. Y. Yao, and C. Monroe, Verified Quantum Information Scrambling, arXiv:1806.02807. 